\title{
Communicability in temporal networks
}

\author{
Ernesto Estrada \\ Department of Mathematics and Statistics, Institute of Complex Systems, University of Strathclyde, 26 Richmond Street, \\ Glasgow G11HX, United Kingdom
}

(Received 3 June 2013; revised manuscript received 9 August 2013; published 17 October 2013)

\begin{abstract}
A first-principles approach to quantify the communicability between pairs of nodes in temporal networks is proposed. It corresponds to the imaginary-time propagator of a quantum random walk in the temporal network, which accounts for unique structural and temporal characteristics of both streaming and nonstreaming temporal networks. The influence of the system's temperature on the perdurability of information and how the communicability identifies patterns of communication hidden in the temporal and topological structure of the networks are also studied for synthetic and real-world systems.
\end{abstract}

DOI: 10.1103/PhysRevE.88.042811

PACS number(s): 89.75.Hc, 02.10.Ox, 05.40.Fb

\section{INTRODUCTION}

Complex networks are frequently considered as static snapshots of the interactions between the elements of a complex system frozen at a given time frame [1-5]. However, these systems are constantly evolving in a way in which both the elements of the system and their interactions change in time, forming the so-called temporal networks [6]. The importance of considering such time-varying complexity of networks has been stressed in recent works [7]. Some of these theoretical frameworks have been already proposed to deal with the ways in which the time-changing topology of a system influences network functioning [7-18]. In particular the use of walks, defined as sequences of (not necessarily different) nodes and links in the network, have found a place in the analysis of temporal networks. However, these walk-based approaches have been developed in an ad hoc way or are based on combinatorial constructions more than on physically sound principles. One of these approaches [19] proposes the use of a Katz-like scheme based on the product of resolvents of the adjacency matrix: $\left(I-\alpha A^{[1]}\right)^{-1}\left(I-\alpha A^{[2]}\right)^{-1} \ldots\left(I-\alpha A^{[h]}\right)^{-1}$, where $I$ is the identity matrix and $\alpha$ is an empirical parameter which depends implicitly on the structure of the network [19-21]. A variation of this method was recently proposed [22], where a Boolean product of matrices is used to account for paths instead of walks.

Here, we propose a first-principles approach to account for the way in which the communicability [23,24] between nodes in a networked system is extended to the time-varying evolution of the system. In this approach we construct the imaginary time-evolution operator of a network which is evolving in time by considering a time-dependent Hamiltonian which is piecewise constant in time. This function accounts for the way in which a perturbation in a node at a given time propagates to another node at another different time. We study here the temporal communicability in both streaming and nonstreaming temporal networks. We use the concept of network temperature for modeling the effect of the level of stress on the links in a network evolving in time.

\section{TEMPORAL COMMUNICABILITY}

We consider here networks represented by (directed or undirected) graphs $H=(V, E)$, with $|V|=n$ nodes and
$|E|=m$ edges. We use a tight-binding Hamiltonian in which each site corresponds to a network vertex $[25,26]$, such that

$$
H(t)=-\sum_{i, j}^{n} \tilde{A}_{i j}(t) c_{i}^{\dagger} c_{j},
$$

where $\tilde{A}_{i j}(t)=1$ if there is a directed link from node $j$ to node $i$ and $\tilde{A}_{i j}(t)=0$ otherwise. Notice that we have used the transpose of the usual adjacency matrix for reasons that will become clear in the next paragraph. If the network is undirected then the adjacency matrix is the usual one. $c_{i}^{\dagger}$ and $c_{j}$ are bosonic or (spinless) fermionic creation and annihilation operators. We consider that the matrix $\tilde{A}(t)$ is piecewise constant in time so that it may be expressed as a composite of the steady operators $\tilde{A}^{[t]}[27,28]$ :

$$
\tilde{A}(t)= \begin{cases}\tilde{A}^{[1]}, & 0 \leqslant t<t_{1}, \\ \tilde{A}^{[2]}, & t_{1} \leqslant t<t_{2}, \\ \vdots & \vdots \\ \tilde{A}^{[h]}, & t_{r-1} \leqslant t<t_{r}=\tau .\end{cases}
$$

Then, the time-evolution operator for a quantum random walk (QRW) [29] in a network with time-evolving topology is defined in the usual way in time-reverse order as

$$
\begin{aligned}
\tilde{G}= & \exp \left(i\left(t_{h}-t_{h-1}\right) \tilde{A}^{[h]}\right) \exp \left(i\left(t_{h-1}-t_{h-2}\right) \tilde{A}^{[h-1]}\right) \cdots \\
& \times \exp \left(i\left(t_{2}-t_{1}\right) \tilde{A}^{[2]}\right) \exp \left(i t_{1} \tilde{A}^{[1]}\right) .
\end{aligned}
$$

We now obtain the communicability function on the temporal network for which the temporal walks go from initial to final times. We first consider that all the time intervals are of the same size and we then use the Wick rotation in order to obtain the imaginary time propagator as $\quad \tilde{G}=\exp \left(\beta \tilde{A}^{[h]}\right) \exp \left(\beta \tilde{A}^{[h-1]}\right) \ldots \exp \left(\beta \tilde{A}^{[2]}\right) \exp \left(\beta \tilde{A}^{[1]}\right)$, where $\beta=\left(k_{B} T\right)^{-1}$ is the inverse temperature. Now, by using the properties of the transpose of the product of matrices as well as those of the transpose of the matrix exponential we obtain the communicability matrix for a network as

$$
G=\tilde{G}^{T}=\exp \left(\beta A^{[1]}\right) \exp \left(\beta A^{[2]}\right) \ldots \exp \left(\beta A^{[h]}\right) .
$$

Obviously, if $A^{[t]}=0$ for all $t \neq k$, then $G=\exp \left(\beta A^{[k]}\right)$, which is the network communicability or thermal Green's function of the network previously studied in the literature [24]. Also notice the difference between the inverse 
temperature, which does not depend on the structure of the network, and the empirical parameter $\alpha$ in the Katz-like measure [19-21], which depends implicitly on the structure of the network via the largest eigenvalue of the adjacency matrix. The temperature here measures the level of stress to which the links of the network are submitted. The hightemperature limit $\beta \rightarrow 0$ indicates a high level of stress in the links which decreases the communication through them to almost zero. This situation models what happens in the real world when there is a high level of social agitation in socioeconomic networks, a biological system is submitted to extreme physiological conditions, or some sort of physical overexploitation is applied to engineering or infrastructural networks.

The expression for the temporal communicability $G$ combinatorially means that we penalize the walks occurring in the same time frame more heavily than those occurring across different times. That is, a walk $\mu_{k}$ of length $k$ that starts and ends at time $t_{i}$ is penalized by $k !^{-1}$. However, a walk $\mu_{k}$ of total length $k$ that "travels" through the times $t_{i}-t_{i+1}$ $-\cdots-t_{j} \quad$ with lengths $l\left(t_{i}\right), l\left(t_{i+1}\right), \ldots, l\left(t_{j}\right)$, such that $k=l\left(t_{i}\right)+l\left(t_{i+1}\right)+\cdots+l\left(t_{j}\right), \quad$ is penalized by $\left(l\left(t_{i}\right) ! l\left(t_{i+1}\right) ! \cdots l\left(t_{j}\right) !\right)^{-1}$, which is smaller than $k !^{-1}$. This penalization scheme implies that the temporal communicability (4) favors the transmission of information through time more than the communication between nodes in the same time frame. As a simple example let us consider the following case. Suppose that there is a walk of length $k$ which diffuses through the nodes of a network only at time $t_{i}$. Then, when $k \rightarrow \infty$, the contribution of this walk tends to zero. However, a walk of length $k$ which has only one step at each time from $t_{i}$ to $t_{j}$ is just divided by 1 ! as it will correspond to the case $A_{i} A_{i+1} \cdots A_{j}$, favoring very much the transmission of the information from the initial to the final time frame. In the case of the Katz-like communicability [16] this walk is heavily penalized, implying that its contribution goes to zero as $k \rightarrow \infty$, which indicates that there is absolutely no transmission of information from the first to the last time frames.

In order to quantify the total amounts of communicability broadcasted and received by a given node in the total process of communication in time we follow the proposal in Ref. [16] of taking the sum of the columns and rows of the $G$ matrix as the broadcast and receive centralities, respectively:

$$
G_{p}^{\text {broadcast }}=\sum_{i=1}^{n} G_{p i}, \quad \text { and } \quad G_{p}^{\text {receive }}=\sum_{i=1}^{n} G_{i p} .
$$

The sum of the rows and columns of the static communicability matrix has been recently proposed as a centrality measure, with a few computational advances over the individual communicability terms $G_{p q}$ [30].

\section{TIME-PATHS AND THE EFFECT OF THE TEMPERATURE}

In a temporal network there are some paths that travel through time. That is, a time-path is a sequence of nodes and edges of the form: $v_{i}^{[t]} e_{i j}^{[t]} v_{j}^{[t]} e_{j s}^{[t+1]} v_{s}^{[t+1]} \cdots v_{p}^{[h]} e_{p q}^{[h]} v_{q}^{[h]}$. The simplest scenario in which such time-paths appear is when the temporal network consists of $h$ snapshots each of them consisting of graphs with only one edge, such that if there is an edge between nodes $i$ and $j$ at time $t$, there is also an edge between $j$ and any node $k$ at time $t+1$. For instance, we can consider adjacency matrices of the form

$$
A_{i j}^{(t)}=A_{j i}^{(t)}= \begin{cases}1 & \text { if } i=t \text { and } j=i+1, t=1 \cdots h \\ 0 & \text { otherwise. }\end{cases}
$$

In this case the temporal communicability function $G=$ $\exp \left(A^{[1]}\right) \exp \left(A^{[2]}\right) \cdots \exp \left(A^{[h]}\right)$ can be written as

$$
G=\left(\begin{array}{ccccccc}
\alpha & \alpha \delta & \alpha \delta^{2} & \alpha \delta^{3} & \cdots & \alpha \delta^{n-2} & \delta^{n-1} \\
\delta & \alpha^{2} & \alpha^{2} \delta & \alpha^{2} \delta^{2} & \cdots & \alpha^{2} \delta^{n-3} & \alpha \delta^{n-2} \\
0 & \delta & \alpha^{2} & \alpha^{2} \delta & \cdots & \alpha^{2} \delta^{n-4} & \alpha \delta^{n-3} \\
0 & 0 & \delta & \alpha^{2} & \cdots & \alpha^{2} \delta^{n-5} & \alpha \delta^{n-4} \\
\vdots & \vdots & 0 & \delta & \cdots & \alpha^{2} \delta^{n-6} & \alpha \delta^{n-5} \\
0 & 0 & \vdots & \vdots & \ddots & \vdots & \vdots \\
0 & 0 & 0 & 0 & \cdots & \delta & \alpha
\end{array}\right),
$$

where $\alpha=\cosh (\beta)$ and $\delta=\sinh (\beta)$. As can be seen, although the individual communicability matrices for individual time snapshots could be very sparse, the total temporal communicability matrix is denser. For instance, in the example studied all individual communicability matrices, i.e., $G^{[t]}=$ $\exp \left(A^{[t]}\right), t=1, \ldots, h$, are very sparse with only a couple of nondiagonal entries different from zero. However, as can be seen in Eq. (7) the temporal communicability matrix can have all the upper triangular entries different from zero.

As a consequence of Eq. (7) the communicability between any pairs of nodes depends only on the inverse temperature $\beta$. Before proceeding it is time to dedicate some words to the meaning of the temperature in this context. The term "temperature" is used here as a quantifier of the external stress to which a complex network is submitted. By external we mean an effect which is independent of the topology of the network. For instance, in a social network the temperature accounts for the different levels of social agitation in the network, in economic networks it accounts for the level of economic stress ranging from bonanzas to economic crisis and in biological networks the temperature can account for the level of physiological stress. We can account for such temperature effects if we consider that the complex network is submerged into a thermal bath at the temperature $T$. The thermal bath represents the external situation which affects all the links in the network at the same time. After equilibration all links in the network are weighted by the parameter $\beta=\left(k_{B} T\right)^{-1}$, where $k_{B}$ is the Boltzmann constant.

Let us now continue with our analysis of the effect of the temperature over the communicability in a temporal path. According to Eq. (7), for $\beta<\ln (1+\sqrt{2}) \approx 0.8814$, the value of $\delta<1$, which means that the communicability between a pair of nodes decays with their separation in the time-path. That is, as the information passes through time it is degrading such that the communicability between distant nodes in time is negligible. On the other hand, if $\beta>\ln (1+\sqrt{2}) \approx 0.8814$ the communicability increases with the separation of the nodes, 

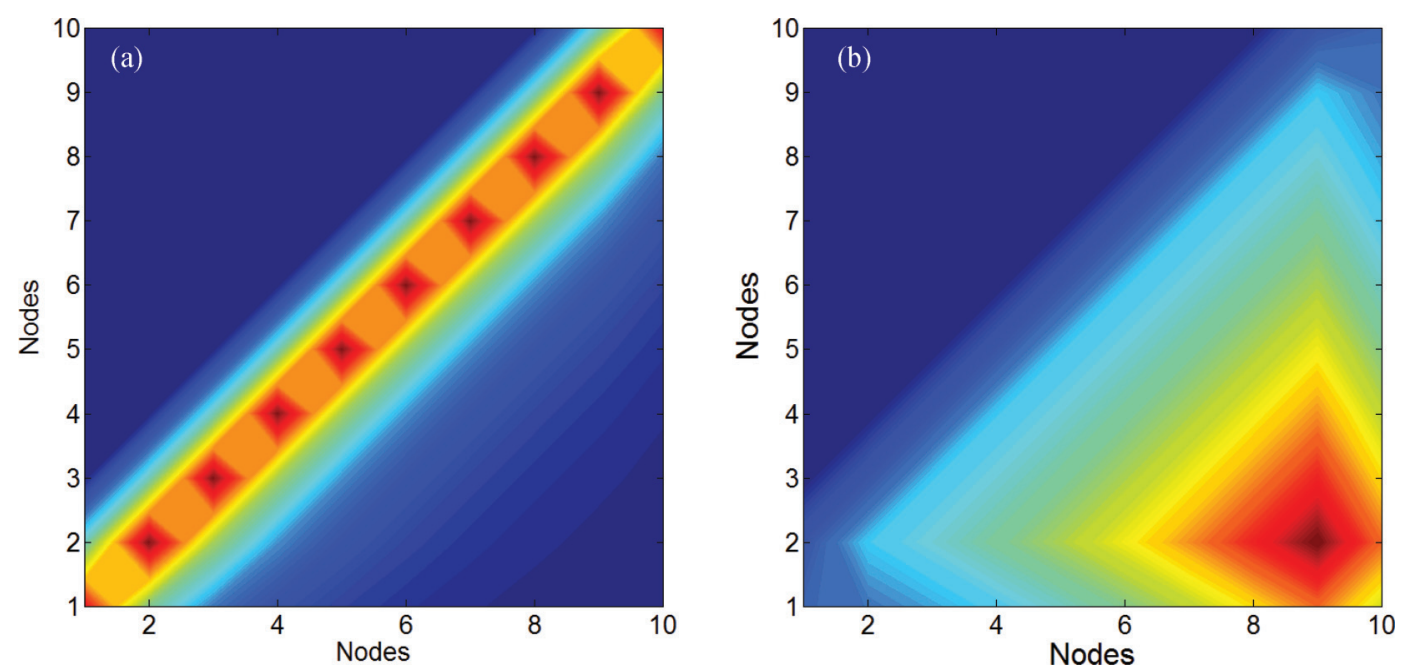

FIG. 1. (Color online) Contour plot of the temporal communicability between pairs of nodes in a temporal path of ten nodes and nine time steps for two different temperatures: $\beta=0.5$ (a) and $\beta=1.0$ (b).

indicating that there is an amplification of the information while traveling through the time-path. This is illustrated in Fig. 1 where the communicability between every pair of nodes is plotted for a time-path of ten nodes and nine time steps. As can be seen when $\beta<\ln (1+\sqrt{2}) \approx 0.8814$ [Fig. 1(a)] all the communicability is concentrated between the pairs of nodes which are very close in time. However, for $\beta>$ $\ln (1+\sqrt{2}) \approx 0.8814$ [Fig. 1(b)] the largest communicability is observed for pairs of nodes which are distant in time, indicating an amplification of the communication as the time passes.

\section{TEMPORALLY STREAMING NETWORKS}

We start by considering a synthetic streaming temporal network. The total (aggregate) network consists of 1000 nodes and 1250 links. The temporal network is formed by 12 time frames in each of which there are 100 nodes connected randomly by 100 links. The last time frame consists only of 50 nodes. We consider that $20 \%$ of the nodes randomly interacting at time $t-1$ can also interact randomly at time $t$, producing an overlapping in the communication between the groups. Using this randomly generated streaming network we study the time-evolving communicability between every pair of nodes.

In Fig. 2(a) we illustrate the temporal communicability for all the pairs of nodes in the streaming network. As can be seen the largest time-varying communicability occurs between some of the nodes in the very first group, i.e., those interacting at time $t=1$, and some of the nodes appearing at the very last group, i.e., those appearing at time $t=13$. The highest broadcasters of the systems are the nodes of the first group and the highest receivers are those appearing at the end. The first group does not show high receive communicability as they have not been "communicated" by any other group. Also, the last group is a bad broadcaster as it does not have a next group to communicate with. Consequently, a negative correlation is observed between $G_{p}^{\text {broadcast }}$ and $G_{p}^{\text {receive }}$ as can be seen in Fig. 2(b). These results are very much in contrast with those obtained by the Katz-like time-varying communicability [see Fig. 2(c)]. In this case all communication occurs between the nodes in the same group (the same time frame) and no cumulative effect of the time-varying communicability is observed. That is, the best broadcasters are also the best receivers as they belong to the same group in the time stream. This situation resembles the case when the information dies out quickly in time, in such a way that there is almost no transmission to further "generations" of nodes.

The second situation discussed in the previous paragraph where the information dies out quickly in time can also be accounted for by Eq. (4) by considering the limit $\beta \rightarrow 0$. It is straightforward to realize that in this case $G \rightarrow I$, where $I$ is the identity matrix of the corresponding size. That is, at very high temperatures there is no communicability between any pair of nodes. As can be seen in Fig. 2(d), when the temperature increases, the temporal communicability between nodes distant in time decays to zero and the only remaining communication is among the nodes in the same temporal frame. In other words, the temperature acts as a controller for the temporal transmission of information. When the temperature is low there are better chances for the information to be transmitted through longer time frames than when $\beta \rightarrow 0$.

The situation described before models several types of networked systems evolving in a time-streaming way in the real-world, such as visitors and customs in galleries, museums, shops, cafes, transport services, among others. In these cases the time-varying communicability function (4) captures the cumulative effect in time of the information (a comment, rumor, gossip, or even a viral charge) placed by the groups arriving first in time. These cumulative effects are the main cause of perdurable stories that can become knowledge or myths with time. In order to study the communicability in a real-world streaming time-varying scenario we consider here a network of deployments at the Science Gallery in Dublin (http://www.sciencegallery.com/infectious) studied for a period of 3 months. In this study the visitors were equipped 

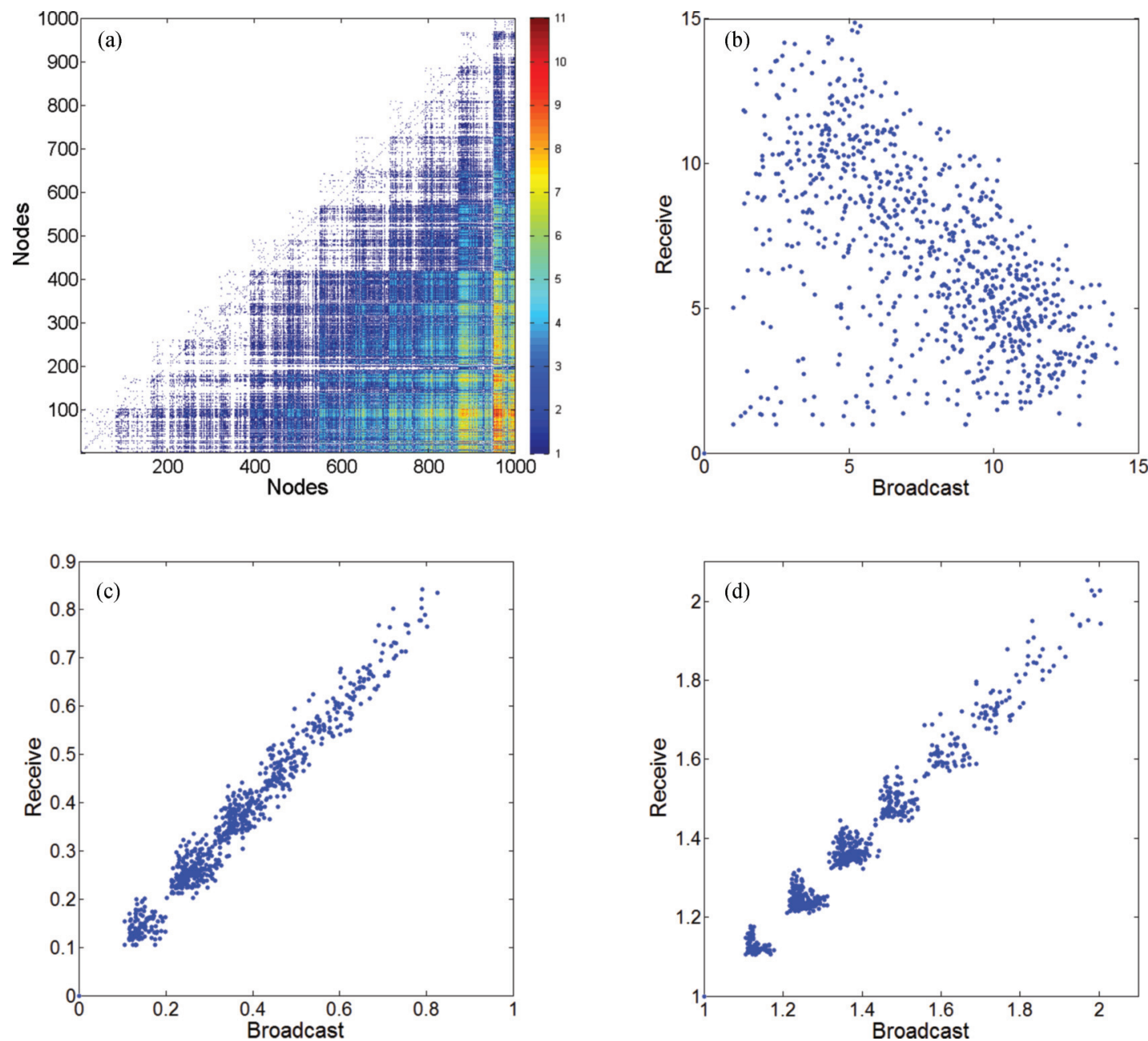

FIG. 2. (Color online) Communicability for every pair of nodes (a) and scatterplot of the broadcast versus receive centrality for a synthetic streaming temporal network with 1000 nodes and 1250 edges: (b) using Eq. (4) of this work using $\beta=1$, (c) Katz-like communicability [16], and (d) using Eq. (4) of this work using $\beta=0.1$. The values of communicability in all plots are in logarithmic scale.

with a radio-frequency identification tag at the entrance of the Gallery to track their locations. Two persons are considered to be interacting if there is an exchange of radio packets between their badges, which is only possible if they are in a range between 1 and $1.5 \mathrm{~m}$ and facing each other (see Ref. [7] and references therein for more details). As can be seen in Fig. 3 the communicability patterns for this network display the signature of time-streaming networks for $\beta=1$, which is characterized by a very large asymmetry. In this network the information is amplified from the broadcasters, who are the visitors in the groups arriving at early times, to the receivers, who are those arriving at later times. In contrast, the communicability among the visitors sharing the same time frames in the Gallery is relatively small and it is only observed by the spikes close to the main diagonal of Fig. 3 (left). These two characteristics are reflected in the strongly negative correlation between the broadcast versus receive communicability for all the nodes in the network (see Fig. 3). In this case it is clear that the main broadcasters are quite different from the main receivers as they are far apart in time in the network. These characteristics are observed neither by the analysis of the aggregate network nor by the use of resolventlike communicability functions. We also have seen in this network that the increase of the temperature, i.e., $\beta \rightarrow 0$ (data not shown), changes dramatically the shape of the communicability patterns in the network in a way similar to that observed for the synthetic network previously analyzed. In this case, the increase of the temperature implies that the long-time transmission of information decays almost to zero and all communicability is mainly concentrated on individuals visiting the Gallery in the same time frame.

\section{TEMPORALLY NONSTREAMING NETWORKS}

We now explore the characteristic features of the temporal communicability function for nonstreaming networks. In this case we study two datasets, the first concerning email activities of Enron employees [31] and the second consisting of a network of "who phoned who" between 106 individuals based at M. I. T. over 365 days [32]. The first network consists of daily information transferred via email between 151 Enron employees over 1138 days starting on May 11, 1999. This network is directed due to the fact that email communication can be unidirectional. Despite that the telephonic communication 

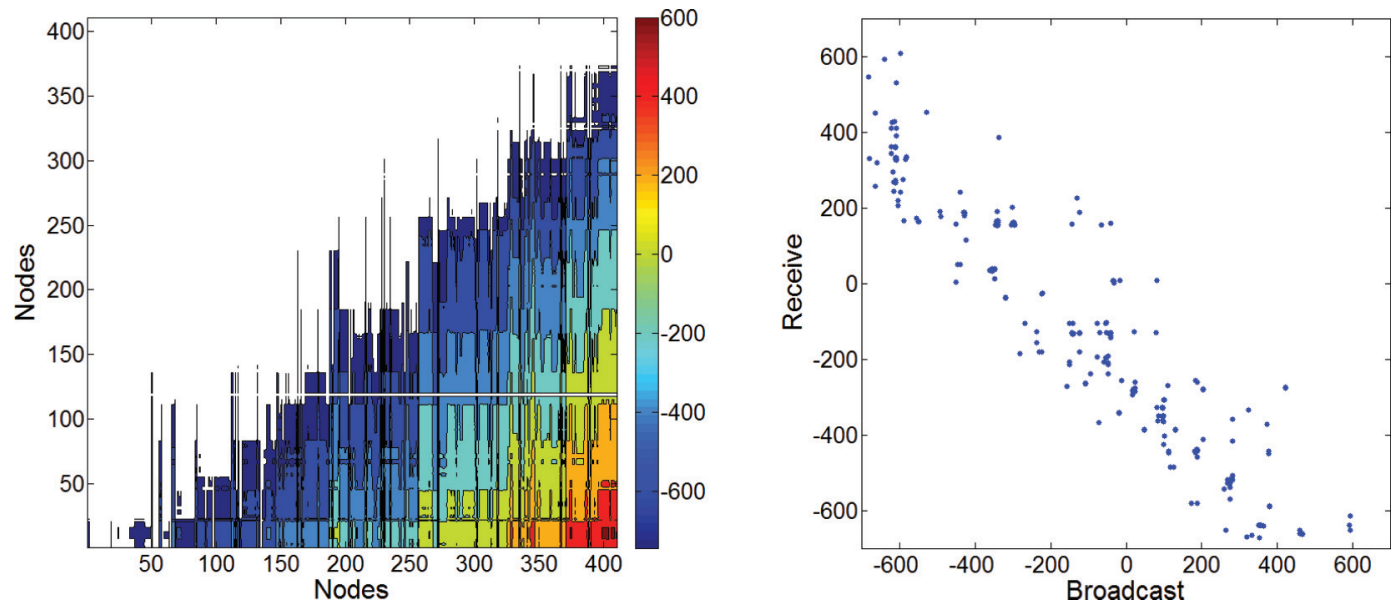

FIG. 3. (Color online) Interperson temporal communicability for the 410 agents in the socio pattern study in the Science Gallery in Dublin. Using Eq. (4) every exponential matrix was divided by $10^{45}$ in order to avoid huge numbers and the communicability was taken in logarithmic scale.

is bidirectional we consider here a directed network for the case of the M. I. T dataset due to the fact that the data accounts for who phoned who, which indicates who are the people that initiate the communication.

In the cases of nonstreaming temporal networks, the temporal communicability does not display the typical patterns that we have observed previously for streaming networks, but rather a characteristic one of networks in which communication flows in a more regular basis. For instance, the difference between the information broadcasted and received, $G_{p q}^{\text {broadcast }}-G_{p q}^{\text {receive }}$, for each pair of nodes on the basis of Eq. (4) clearly indicates that a few employees displayed an odd pattern of communication with significantly larger information (email) broadcasted than received. The same pattern is obtained by the analysis of the scatterplot of both centralities in Fig. 4(a). Among these high broadcasters with low receiving patterns it is easy to recognize some of the main players in the Enron scandal, whose patterns of communication are totally hidden if the analysis is focused on the aggregate network. In the aggregate network a quite strong correlation is observed between the broadcast and receive communicability as can be seen in Fig. 4(b).

Finally, we study the network of "who phoned who" between 106 individuals based at M. I. T. over 365 days. In this case we are interested in revealing a pattern in the temporal communicability which cannot be observed by using the aggregate network or the resolvent communicability function. In Fig. 5(a) we illustrate the plot of the broadcast versus the receive centrality for the nodes of this network using the temporal communicability function (4). As can be seen in the plot, the nodes of the network are clearly divided into four groups. The two groups along the main diagonal of the plot display some kind of normal behavior. They correspond to those nodes which have large (low) broadcast communicability and at the same time display large (low) receive communicability. However, the two groups out of the main diagonal display some kind of "anomalous" pattern of communication. One of them (top left corner) displays large receive communicability with low broadcast. They are mainly sinks of the information in terms of temporal communication.
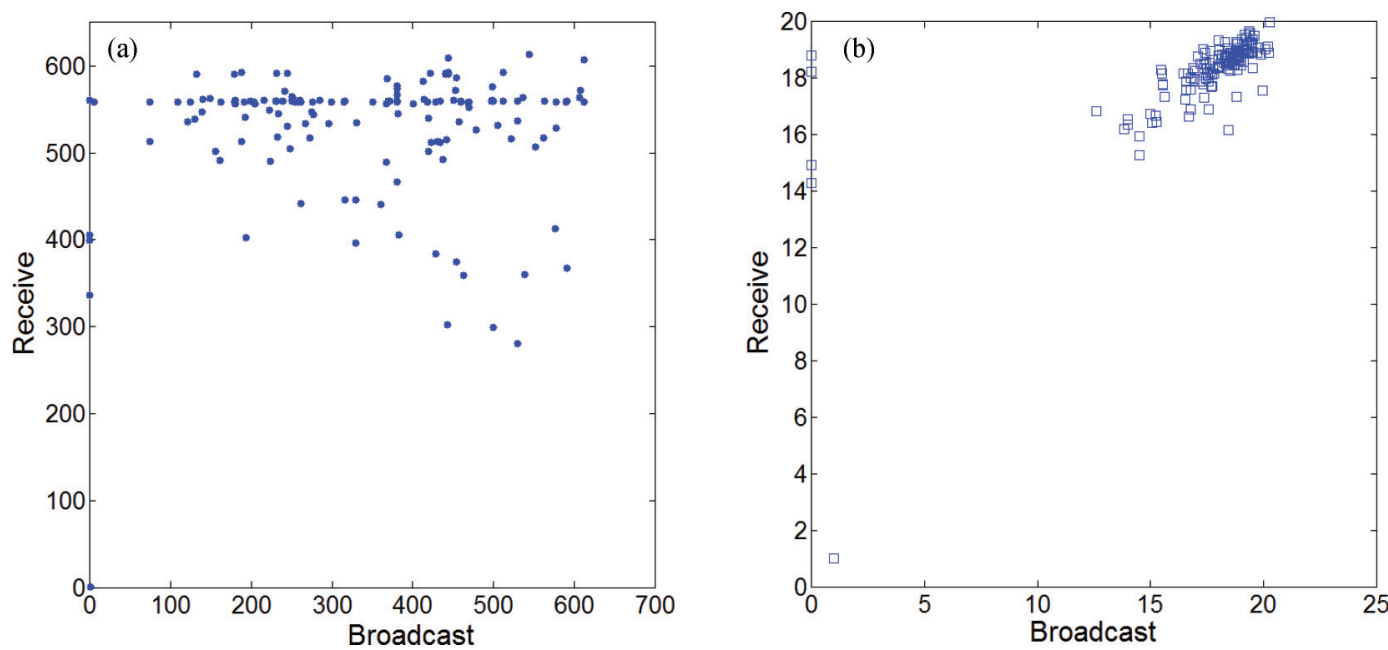

FIG. 4. (Color online) Scatterplot of the broadcast versus receive centrality for the nodes in the Enron dataset using temporal communicability (a) and the aggregate network (b). The values of communicability in all plots are in logarithmic scale. 

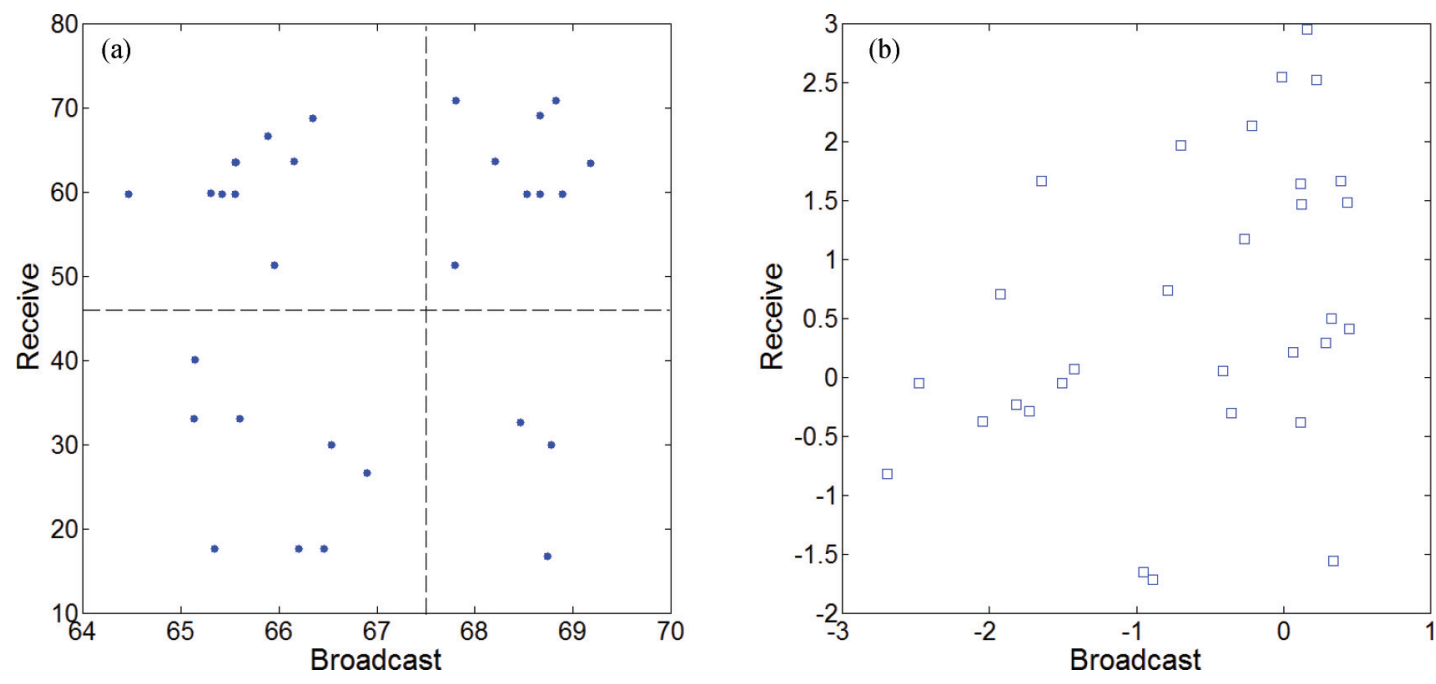

FIG. 5. (Color online) Broadcast versus receive centralities based on the temporal communicability defined in this work (a) and on the Katz-like model (b) of Ref. [19] for the nodes in the M. I. T. phone network. The values of communicability in all plots are in logarithmic scale.

The other group (bottom right corner) displays high broadcast with low receive communicability and represents net spreaders in the temporal communication network. Neither of these groups can be detected by using the temporal resolventlike communicability [Fig. 5(b)] or the aggregate network (plot not shown), which indicates that the temporal communicability based on Eq. (4) makes a good contribution to the analysis of temporal patterns in time-evolving networks.

\section{CONCLUSIONS}

In this work we have proposed a new theoretical method of quantifying how much information flows through the nodes of a network which changes in time. This theoretical frame constitutes a natural extension to temporal networks of the communicability function widely studied for static complex networks. The measure accounts for the imaginarytime propagator of the temporal network, whose evolution is controlled by a time-varying Hamiltonian which is piecewise constant in time. Using this function we have studied streaming and nonstreaming temporal networks, showing that there are significant and relevant differences in the way in which information flows in these two different scenarios. We use the temperature as a measure of the level of stress to which the links of the networks are submitted. In a streaming network, information is preferentially transmitted from the groups arriving first in time to those arriving at later times if the temperature of the system is relatively low. As soon as the temperature grows, the transmission of information through time deteriorates and the only possible transmission is through the members of the groups which communicate among them in the same time frame. In the cases of nonstreaming temporal networks, the temporal communicability brings possibilities for identifying patterns of communication and behaviors which are hidden in the temporal and topological structure of the networks. In closing, the definition and study of the temporal communicability measure represents a valuable contribution to the development of the most needed theoretical frameworks to deal with temporal networks appropriately in a context of physically sound concepts.

\section{ACKNOWLEDGMENTS}

The author thanks N. Hatano and M. Benzi for useful comments on a preliminary version of this work. He also thanks the EPSRC and the RCUK Digital Economy program for support through the project Mathematics of Large Technological Evolving Networks (MOLTEN).
[1] S. Boccaletti, V. Latora, Y. Moreno, M. Chavez, and D.-U. Hwang, Phys. Rep. 424, 175 (2006).

[2] L. de F. Costa, F. A. Rodrigues, G. Travieso, and P. R. Villas Boas, Adv. Phys. 56, 167 (2007).

[3] A. Barrat, M. Barthélemy, and A. Vespignani, Dynamical Processes on Complex Networks (Cambridge University Press, Cambridge, UK, 2008).

[4] M. E. J. Newman, Networks: An Introduction (Oxford University Press, Oxford, 2010).

[5] E. Estrada, The Structure of Complex Networks: Theory and Applications (Oxford University Press, Oxford, 2011).

[6] P. Holme and J. Saramäki, Phys. Rep. 519, 97 (2012).
[7] J. Stehlé, A. Barrat, and G. Bianconi, Phys. Rev. E 81, 035101(R) (2010)

[8] L. Isella, J. Stehlé, A. Barrat, C. Cattuto, J. F. Pinton, and W. Van den Broeck, J. Theor. Biol. 271, 166 (2011).

[9] J. P. Onnela, J. Saramäki, J. Hyvönen, G. Szabó, D. Lazer, K. Kaski, J. Kertész, and A.-L. Barabási, Proc. Natl. Acad. Sci. U.S.A. 104, 7332 (2007).

[10] J. Tang, S. Scellato, M. Musolesi, C. Mascolo, and V. Latora, Phys. Rev. E 81, 055101 (2010).

[11] K. Zhao, M. Karsai, and G. Bianconi, PLOS ONE 6, e28116 (2011). 
[12] P. Bajardi, A. Barrat, F. Natale, L. Savini, and V. Colizza, PLOS ONE 6, e19869 (2011).

[13] G. Miritello, E. Moro, and R. Lara, Phys. Rev. E 83, 045102 (2011).

[14] R. K. Pan and J. Saramäki, Phys. Rev. E 84, 016105 (2011).

[15] K. Zhao, J. Stehlé, G. Bianconi, and A. Barrat, Phys. Rev. E 83, 056109 (2011).

[16] M. Starnini, A. Baronchelli, A. Barrat, and R. Pastor-Satorras, Phys. Rev. E 85, 056115 (2012).

[17] A. Barrat, B. Fernandez, K. K. Lin, and L.-S. Young, Phys. Rev. Lett 110, 158702 (2013).

[18] Z. Q. Jiang, W. J. Xie, M. X. Li, B. Podobnik, W. X. Zhou, and H. E. Stanley, Proc. Natl. Acad. Sci. U.S.A 110, 1600 (2013).

[19] P. Grindrod, M. C. Parsons, D. J. Higham, and E. Estrada, Phys. Rev. E 83, 046120 (2011).

[20] P. Laflin, A. V. Mantzaris, F. Ainley, A. Otley, P. Grindrod, and D. J. Higham, Lect. Notes Comput. Sci. 7710, 82 (2012).

[21] P. Grindrod and D. J. Higham, SIAM Rev. 55, 118 (2013).
[22] H. H. K. Lentz, T. Selhorst, and I. M. Sokolov, Phys. Rev. Lett. 110, 118701 (2013).

[23] E. Estrada and N. Hatano, Phys. Rev. E 77, 036111 (2008).

[24] E. Estrada, N. Hatano, and M. Benzi, Phys. Rep. 514, 89 (2012).

[25] E. Estrada and N. Hatano, Chem. Phys. Lett. 439, 247 (2007).

[26] K. Rudinger, J. K. Gamble, M. Wellons, E. Bach, M. Friesen, R. Joynt, and S. N. Coppersmith, Phys. Rev. A 86, 022334 (2012).

[27] D. R. Lester, M. Rudman, G. Metcalfe, and H. M. Blackburn, J. Comput. Phys. 227, 3032 (2008).

[28] T. Ichikawa, M. Bando, Y. Kondo, and M. Nakahara, Phys. Rev. A 84, 062311 (2011).

[29] J. Kempe, Contemp. Phys. 44, 307 (2003).

[30] M. Benzi and C. Klymko, J. Complex Networks (2013), doi: 10.1093/comnet/cnt007.

[31] N. Eagle, A. S. Pentland, and D. Lazer, Proc. Natl. Acad. Sci. U.S.A 106, 15274 (2009).

[32] A. Chapanond, M. Krishnamoorthy, and B. Yener, Comput. Math. Org. Theor. 11, 265 (2005). 